\title{
La Cognition située sous le regard du paradigme historico-culturel vygotskien ${ }^{1}$
}

\section{Christiane Moro}

Le propos de cet article est de mettre en évidence quelques-uns des espaces de discussion théorique qui émergent des travaux de la Cognition située. Pour ce faire, l'auteure effectue dans un premier temps un sondage au sein de certains des travaux développés par les pionniers de cette tradition en tentant de dégager les options théoriques qui s'y déploient. Dans un second temps, l'auteure examine, au travers de trois thématiques-clés abordées dans ces travaux (la problématique des unités d'analyse; la définition de la médiation; la spécificité de l'activité scolaire versus extra-scolaire) le degré de recoupement entre les visées des deux champs théoriques que constituent la Cognition située et le paradigme historico-culturel vygotskien.

Durant les années quatre-vingts (Kirshner \& Whitson, 1997a), différentes voix se font entendre outre-Atlantique, nombre d'entre elles émanant de la scène psychologique éducationnelle sans s'y limiter cependant, qui toutes concourent à élaborer une réflexion renouvelée sur la nature sociale de la cognition et des apprentissages et à réorienter les conceptions éducatives alors fortement imprégnées par la tradition individualiste cognitiviste.

Ces différentes démarches se réunissent autour de l'idée que la construction de la cognition ne peut être envisagée que «située». Autour de ce «label» fédérateur, naît le courant de la Cognition située (situated cognition, cf. par exemple Resnick, 1987, 1994), dénommé aussi «apprentissage situé» (situated learning, $c f$. par exemple, Lave \& Wenger, 1991) qui en français apparaît parfois sous la dénomination d'»apprentissage en contexte» comme le signale Allal (2000, p. 171). Ce courant est généralement conçu comme co-définissant d'une conception "distribuée» de la cognition (distributed cognition, cf. Pea, 1991, 1993; Perkins, 1995). En posant la nature «située/distribuée» de la cognition, ces différentes approches soulignent le rôle instituant de la culture et des formations sociales dans l'apprentissage et dans la construction des connaissances. Constituant un corps grandissant de recherches en sciences humaines, la Cognition située reformule la 
conception de l'apprentissage en un sens radicalement environnementaliste (Anderson, Reder \& Simon, 1997). A une conception de l'apprentissage et de la connaissance qui résulterait de l'expérience de sujets solitaires dans un monde stable et objectif, cette nouvelle conception substitue celle d'un apprentissage et de savoirs résultant de la participation active des sujets apprenants aux contextes sociaux et matériels qui les entourent, la personne et le monde étant entendus comme mutuellement constitutifs.

Différentes filiations sont identifiables au sein de ce courant. Nous mentionnerons certaines d'entre elles au fil de notre texte mais signalons d'ores et déjà l'impact de la «deuxième psychologie» et de ses méthodes inspirées de l'anthropologie et de l'ethnologie qui, si l'on se réfere à Wundt et à ses considérations sur la Völkerpsychologie, nécessitait d'être considérée comme de statut égal à la première (la psychologie physiologique), l'accès complet à la pensée humaine ne pouvant se réaliser que sous condition de la synthèse des deux perspectives. La deuxième psychologie axée sur l'étude de l'homme et de sa culture se verra pourtant reléguée au profit de la première dont le modèle inspiré des sciences de la nature et fondé sur la méthode expérimentale s'instituera comme la référence dominante en psychologie au cours du vingtième siècle - la culture dans ce nouveau schéma se voyant alors réduite au rang de simple facteur (Cole, 1996). Aux Etats-Unis, la version (de la deuxième psychologie) qui influencera les travaux de la Cognition située se loge dans le courant pragmatiste hérité de Peirce et de James, notable dans les travaux de Dewey $(1913,1938)$ et de Mead (1934). Ces derniers ont particulièrement insisté sur le caractère complémentaire des processus de socialisation et d'individuation et sur le fait que la source du savoir devait dès lors être recherchée dans les activités quotidiennes, culturellement et historiquement organisées du groupe social. Une autre source d'influence au moins égale à celle indiquée ci-dessus est celle issue de l'Ecole historico-culturelle soviétique de Vygotski-Léontiev-Luria. De nombreuses passerelles théoriques existent entre ces deux traditions. En témoignent les intérêts réciproques des penseurs soviétiques pour la pensée occidentale (européenne et nord-américaine) afférant à la deuxième psychologie qu'ils diffusèrent largement en Union Soviétique, et des courants culturels américains qui favorisèrent notablement l'infusion des idées de l'Ecole historico-culturelle soviétique, notamment vygotskienne, aux EtatsUnis (cf. Cole, 1996).

Le courant de la Cognition située n'est pas isolé. Il se situe dans un Zeitgeist qui en sciences humaines/sociales s'intéresse «aux processus émergents qui ne peuvent se réduire à des structures générales» (Hanks, 1991, p. 16), privilégiant notamment les approches phénoménologiques, interactives et pragmatiques. Ces courants contestataires sont autant manifestes en psychologie, en sociologie qu'en linguistique ou en éducation et posent la nécessité d'une réflexion pluridisciplinaire (Bruner, 1990; Cole, 1996). Ils tendent à insister sur les aspects dy- 
namiques (voire imprédictibles) afférant à la construction de la signification et de la communication versus les aspects prédictibles et "préfabriqués» (en termes de codes, de représentations et de structures) de l'action humaine.

Notre propos ici sera de mettre en évidence quelques-uns des espaces de discussion théorique qui émergent de celles des propositions majeures émanant du courant de la Cognition située. Pour ce faire, nous effectuerons dans un premier temps un sondage au sein de certains des travaux développés par les pionniers de cette tradition en tentant de dégager les options théoriques qui s'y déploient. Dans un second temps, nous examinerons, dans le cadre de thématiques-clés, le degré de recoupement entre les visées des deux champs théoriques que constituent la Cognition située et le paradigme historico-culturel vygotskien.

\section{Sondages au coeur de la cognition située}

Dans une tradition de recherches comparatives en apprentissage, inspirée de l'anthropologie mais aussi des conceptions systémiques développées par l'Ecole de Palo-Alto, Lave (Lave 1977a, 1977b, 1988, 1991, 1997; Lave \& Wenger, 1991) s'intéresse aux pratiques quotidiennes dans le contexte indigène des activités organisées. Nombre de ses travaux ont été réalisés dans des sociétés traditionnelles et en milieu extra-scolaire (cf. le projet Vai et Gola sur l'apprentissage du métier de tailleur au Libéria).

Lave et Wenger (1991) distinguent deux termes en anglais: apprenticeship et learning, que le français indifférencie. Pour comprendre la notion d'apprenticeship, il faut la rapporter à l'activité de l'apprenti qui est "en apprentissage». Cette notion contraste avec le concept d'apprentissage au sens classique du terme (learning) à la connotation plus décontextualisée. La considération de cette forme d'apprentissage qu'est l'apprenticeship conduit les auteurs à examiner l'«apprentissage comme apprentissage situé» (1991, p. 31) qu'ils tentent de capter au travers du concept de "participation périphérique légitime» (legitimate peripheral participation) (p. 31), proposé comme «descripteur d'engagement au sein de la pratique sociale» (p. 35, trad. pers.).

Ce qui est central dans cette approche, ce sont les types d'engagements sociaux qui permettent à l'apprentissage d'advenir, l'acquisition de capacités étant considérée comme se réalisant au cours du processus de participation de l'apprenant à la "communauté de pratiques» et non pas en termes d'acquisition d'un corps de savoir abstrait et décontextualisé qu'il s'agirait ultérieurement d'appliquer en contexte. Une communauté de pratiques consiste en un groupe de gens qui sont reconnus comme ayant une expertise dans une pratique culturelle spécifique, tels les sages-femmes, les enseignants, les Alcooliques Anonymes, etc. Cette notion avancée par Lave \& Wenger bien que largement intuitive ainsi que les auteurs le reconnaissent eux-mêmes (1991, p. 42), réfere au système de rela- 
tions qui organisent les activités entre les personnes (y compris les objets), incluant tout autant les normes, les valeurs, les attitudes que les savoirs.

Les activités dans lesquelles l'apprenant s'engage sont constitutives de l'apprentissage. L'apprentissage se manifeste "par une augmentation de l'accès de l'apprenant à des rôles lui permettant de participer aux performances de l'expert» (Hanks, 1991, p. 17, trad. pers.). Le concept de "périphéralité» (évoqué plus haut) suggère qu'il y a différents modes d'engagement possibles dans la communauté de pratiques. De fait, l'apprentissage n'est pas dissociable du processus de construction identitaire ainsi que l'indiquent les auteurs: «changer de positions et de perspectives fait partie des trajectoires d'apprentissage de l'acteur, du développement d'identités et des formes d'appartenance» (Lave \& Wenger, 1991, p. 36, trad. pers.).

Tout comme Lave, Rogoff, dans ses travaux relatifs au développement de l'enfant, met en avant le caractère intrinsèquement "situé» des processus d'apprentissage et de développement - identifiés l'un à l'autre au sein de sa conception (Rogoff, 1998, p. 680) et insiste également sur le concept d'apprenticeship (Rogoff, 1990). Elle souligne que le développement cognitif advient et est promu dans le cadre de processus de collaboration entre individus dans des contextes institutionnels et communautaires variés. Le processus collaboratif inclut tout type de relation entre les personnes comme le face-à-face, les conversations, le tutorat, l'enseignement, ou l'apprentissage coopératif (Rogoff, 1998) et est étendu aux situations en dehors de la co-présence physique comme entre correspondants ou entre auteurs et lecteurs d'articles (Rogoff \& Toma, 1997, citant Berkowitz \& Gibbs, 1985; Brown \& Palincsar, 1989; Forman \& Cazden, 1985; Johnson \& Johnson, 1989; Mugny, Perret-Clermont \& Doise, 1981).

Rejetant toute forme d'approche qui étudierait le phénomène psychique de manière morcelée, elle indique que la seule voie pour étudier le développement réside dans l'«activité qui inclut les contributions des individus engagés avec d'autres individus, les circonstances matérielles et les traditions culturelles» (Rogoff, 1996, p. 278, trad. pers.). Le développement individuel est capté au travers $\mathrm{du}$ concept de "transformation de participation" ${ }^{2}$ (changing participation) (Rogoff, 1996, 1998) des apprenants aux activités socio-culturelles. Pour approcher les processus interpersonnels et personnels, Rogoff développe le concept de "participation guidée» $(1990,1995,1996,1998)$ qui rend compte des occasions qui sont faites à l'enfant de se diriger vers les activités pertinentes dans les diverses communautés (Rogoff, 1998, p. 700) et corrélativement celui d' «appropriation participatrice» (Rogoff, 1996, p. 279). Ces concepts lui permettent, par exemple, d'examiner la construction des conduites d'autonomie chez l'enfant au sein de diverses communautés (Rogoff, 1996).

Le concept de participation - tout comme chez Lave \& Wenger (1991) - se distingue de l'étayage (scaffolding) (Wood, Bruner \& Ross, 1976) auquel il tend parfois à être assimilé (Rogoff, 1998). Le concept d'étayage est considéré comme 
trop mécanique (Griffin \& Cole, 1984; Valsiner \& Van der Veer, 1993) et induit une conception passive de l'apprentissage (Packer, 1993). En faisant en effet reposer l'essentiel de la "construction» (pour poursuivre la métaphore) chez l'apprenant sur l'activité du tuteur, l'étayage tend à disqualifier l'activité de l'apprenant et sa capacité à infléchir l'interaction et les instructions du tuteur. Dans le même temps, les contextes culturels et institutionnels sont négligés (Rogoff, 1990). L'unité d'analyse se trouve dès lors restreinte à la seule activité du tuteur et aux fonctions qu'elle remplit (Rogoff, 1998).

Certains auteurs examinent plus spécifiquement la question de la nature «distribuée» de la cognition (comme par exemple, Hutchins, 1990, 1991, 1993, 1995; Pea, 1993; Resnick, 1987). Ce concept, déjà présent chez Lave (1988, 1991), est à entendre comme complémentaire voire co-définissant d'une conception située de la cognition. La distribution (voir par exemple, Pea, 1993; Perkins, 1995) réfere au fait que les savoirs ne sont pas seulement logés chez l'«individu solo» (l'expression est de Perkins, 1995) mais sont répartis entre les différents individus et les outils (matériels et symboliques) qui nous entourent et auxquels nous recourons en activité solitaire ou collective. La relation homme-machine (et particulièrement homme-ordinateur) a particulièrement influencé la réflexion sur la nature distribuée de la cognition (Pea, 1991; Salomon, Perkins \& Globerson, 1991). Un champ important de recherches est ainsi né autour de la question des artefacts $^{3}$ et de l'impact de leur utilisation sur l'activité cognitive (cf. entre autres Norman, 1988, 1993). Resnick (1987) cite, comme exemple d'artefact, le compas qui a transformé l'art de la navigation (et donc les tâches cognitives) en suppléant d'abord aux systèmes de repérage par les étoiles et dont la technologie chaque fois plus sophistiquée a modifié et modifie encore l'activité de navigation.

Dans un exemple provenant également de l'activité de navigation, Hutchins (1990, 1993) (voir aussi in Lave \& Wenger, 1991) décrit le travail d'une équipe de six marins de l'U.S. Navy engagés dans le pilotage d'un navire hors du port (avec trois descriptifs de travail différents incluant l'usage d'instruments cognitifs externes complexes en rapport avec la haute technologie du navire). En ce cas, la complexité cognitive de la tâche excède les capacités de l'individu seul, la réussite du pilotage et donc le savoir nécessaire pour piloter le bateau résidant dans le système formé par les personnes (les marins) et les outils techniques (qualifié par Hutchins de système de cognition distribuée).

Hutchins (1997) souligne qu'un nombre important de tâches ordinaires sont médiatisées par une grande diversité d'artefacts considérés comme des «structures médiatisantes qui ne sont pas en elles-mêmes inhérentes au domaine de la tâche» (p. 338, trad. pers.). Ces artefacts peuvent être relativement banaux et ne pas présenter nécessairement un haut degré de sophistication technologique, à l'exemple de la «liste de contrôle». 
Alors que les positions évoquées ci-dessus plaident en faveur d'un savoir s'acquérant et se spécifiant dans le cadre des seules situations sociales et historiques spécifiques, Resnick (1994) propose un modèle d'apprentissage et de développement, qualifié de rationalisme situé (situated rationalism), qui vise à réconcilier perspective biologique et perspective sociale. Cette conception tente plus précisément d'articuler les positions sur le caractère situé/distribué de la cognition développées par Lave et Hutchins (qualifiées par Resnick de situated rationalism) et les conceptions qui postulent la notion de structure comme «unité de la cognition» (p. 475, trad. pers.) telles celles de Gestaltistes comme Wertheimer ou Piaget (qualifiées par Resnick de conceptual rationalism). Une bonne métaphore du fonctionnement cognitif au sein de cette nouvelle configuration se situerait pour Resnick dans les modèles connexionnistes de la cognition ou PDP (Parallel Distributed Processing) de Rumelhart \& McClelland (1986) [également invoqués par Hutchins pour la modélisation des tâches instrumentées]. Dans son modèle, Resnick propose d'étendre l'idée de structures préparées (prepared structures) audelà du biologique et d'y inclure la notion de préparation sociale de façon à théoriser l'apprentissage spécifiquement situé. Ainsi que l'indique Resnick (1994),

les rationalistes conceptuels font valoir que l'apprentissage advient lorsque les structures préparées sont élaborées au cours de l'interaction avec l'environnement. Les structures préparées dirigent et contraignent l'attention vers des faits particuliers de l'environnement qui vont soutenir l'élaboration des concepts particuliers. Ils rendent l'individu sensible aux affordances particulières des situations. Dans notre conception d'une préparation socio-culturelle des structures, il est facile d'imaginer que les structures résultant d'engagements préalables dans le cadre de situations culturelles spécifiques contraignent de manière similaire la façon dont les individus entrent dans de nouvelles situations. En situation d'engagement avec l'environnement, les structures préparées sont modifiées et élaborées par cet engagement. [...] C'est ce processus que nous appelons apprentissage (p. 479, trad. pers.).

La question centrale qu'un tel modèle vise à élucider est celle des relations entre structures biologiques préparées et structures de savoir culturellement élaborées. Ces relations peuvent être cohérentes ou contradictoires avec les structures biologiques selon les savoirs, voire être un mélange des deux comme c'est le cas pour de nombreux concepts proposés à l'apprentissage. Ainsi par exemple, la composition additive des nombres entiers positifs constitue un exemple de compatibilité avec les structures biologiques tandis que les lois newtoniennes du mouvement "contredisent les croyances qui sont enracinées dans les structures biologiques préparées» (p. 481, trad. pers.). Les conséquences que Resnick en tire pour l'éducation sont que, dans le cas de la compatibilité, l'acte d'enseigner consiste essentiellement à apporter une aide aux enfants de manière à faire évoluer les concepts biologiques initiaux vers les formes culturelles adaptées. Dans le cas de l'incompatibilité, où il s'agit d'aider l'enfant à remplacer (plutôt qu'à élaborer) les croyances initiales, les solutions éducatives restent encore à trouver. 
Ces solutions pourraient passer par l'élaboration d'une "théorie des situations" (p. 491, trad. pers.) qui permettrait de dégager les dimensions sociales, cognitives et physiques desdites situations ainsi que de déterminer ce qui dans une situation prépare l'enfant à entrer dans une autre situation. La théorie des situations devrait à terme permettre d'apporter des éléments de réponse à la problématique cruciale du transfert que le paradigme cognitiviste ne résout pas de manière satisfaisante (cf. aussi Greeno, Moore \& Smith, 1993).

Brown, Collins \& Duguid (1989), dans un texte majeur pour l'éducation, donnent un élan déterminant à la réflexion sur l'école et les pratiques d'enseignement. Se situant dans le sillage des réflexions des auteurs évoqués ci-dessus, et donc dans le prolongement de la réflexion initiée sur l'école à partir des travaux sur l'apprentissage dans les situations quotidiennes (apprenticeship), Brown, Collins \& Duguid arguent du fait que «la connaissance est située, qu'elle est en partie un produit de l'activité, du contexte, et de la culture dans laquelle elle est développée et utilisée». (p. 32, trad. pers.) En ce sens, «l'activité dans laquelle le savoir est développé et déployé, n'est pas séparable [...] de l'apprentissage et de la cognition.» (p. 32, trad. pers.) En conséquence, les situations sont considérées comme co-productrices des savoirs au travers de l'activité.

En guise de synthèse intermédiaire à cette brève incursion dans le champ de la Cognition située, nous voudrions souligner que le déplacement de focus vers l'analyse des situations d'apprentissage a constitué ces dernières années aux EtatsUnis l'une des voies de choix qui a permis que soit réintroduite, au plan de la recherche, la culture en tant que procès constitutif de l'apprentissage et de la cognition. Le concept de situation proposé par Dewey en 1938, initialement lié par lui au contexte ${ }^{4}$, s'est ainsi trouvé réactualisé et notamment mis en lien avec l'activité pratique humaine, plus apte à rendre compte des entrelacs de l'expérience réelle que ne pouvait le faire la psychologie cognitive à l'aide des seuls concepts et représentations mentales. Ce retour sur la scène psychologique de l'activité a notamment permis l'éclosion de nouveaux travaux s'intéressant aux processus dits émergents, soit ceux qui n'ont pas de siège en tant qu'ils sont précisément co-extensifs à l'activité collective (cf. Schwartz, 2001, pp. 67-91) et a amplifié un courant de recherches, manifestes dans d'autres secteurs disciplinaires, sur les interactions.

Comme le signalent Kirshner \& Whitson (1997a \& b), le courant de la Cognition située est essentiellement un mouvement en construction et en évolution, traversé de débats et encore à la recherche d'une position intégrée (Lave, 1988, citée par Kirshner \& Whitson (1997a \& b). Les débats concernent autant la problématique des unités d'analyse à même de pouvoir rendre compte de manière unifiée du développement social et individuel (Forman, Minick \& Stone,1993; Lave, 1988) en lien avec la question de trouver de nouvelles ressources théoriques pour cette élaboration (Kirshner \& Whiston, 1997a); que la 
question de localiser la dynamique des apprentissages dans le seul monde de l'expérience quotidienne qui sacrifierait «les opportunités que l'école offre en matière d'activité tournée vers l'abstraction et la réflexion" (p. viii, trad. pers.). Notre deuxième partie axée sur le questionnement des positions de la Cognition située à l'aide du paradigme historico-culturel vygotskien reviendra sur ces différentes questions.

\section{Gros plan sur trois thématiques-clé de la cognition située et éclairage vygotskien en contrechamp}

L'apprentissage saisi au travers des positions situées/distribuées de la cognition met en avant différents champs de problématiques afférant à quelques-unes des grandes thématiques développées par Vygotski, auteur auquel les tenants de ces conceptions font souvent référence. Un examen plus approfondi de ces thématiques révèle toutefois des angles d'attaque théoriques nuancés voire parfois notoirement divergents entre les modèles de la Cognition située et le paradigme historico-culturel vygotskien. Considérant que ces éclairages constituent un puissant révélateur des positions épistémologiques sous-jacentes aux différents modèles, nous nous proposons d'examiner dans le cadre de notre deuxième partie trois thématiques transversales majeures. Elles concernent la définition des unités d'analyse susceptibles de rendre compte de la construction et du fonctionnement psychiques; la médiation au travers du rôle respectif des outils de la culture et d'autrui dans les processus d'apprentissage-développement; et la spécificité du développement au plan scolaire (versus les activités extra-scolaires dont les travaux sur la Cognition située font majoritairement état). Pour ce faire, il convient que nous rappelions au préalable les grandes lignes du projet théorique vygotskien.

Vygotski défend la thèse de la genèse sociale des fonctions psychiques supérieures 5 faisant valoir que le développement de la pensée et de la conscience, loin d'être une émanation de processus endogènes, résulte fondamentalement de la transformation du fonctionnement psychique comme suite de l'intégration des différentes catégories d'outils forgés par la culture. Sous cette considération, Vygotski propose une approche renouvelée des relations entre apprentissage et développement, l'apprentissage ${ }^{6}$ précédant le développement. L'apprentissage consiste dès lors prioritairement (mais non exclusivement) en l'appropriation effective de la culture dans des contextes communicatifs, l'activité du sujet apprenant dans le monde ne devenant significative que sous l'influence d'autrui. Dans ce cadre, l'éducation - notamment formelle et scolaire - se trouve considérablement revalorisée (cf. le concept de zone proximale du développement» - dit Zoped dans la suite de ce texte ${ }^{7}$. 
Le maillon central de l'approche vygotskienne repose sur le concept de médiation, inspiré des conceptions de Marx et de Engels concernant le rôle joué par le travail et la relation que l'homme établit avec la nature, que Vygostski transpose à la sphère de l'activité psychique et dont deux formulations sont attestables au sein de la théorie vygotskienne (Moro, à paraittre; Moro \& Schneuwly, 1997, pp. 1-17; Schneuwly, 1999, pp. 267-280). Vygotski propose tout d'abord l'unité d'analyse d' «acte instrumental» et développe l'idée d'outils ou d'instruments psychologiques qui, intégrés à la conduite, permettent non seulement d'en modifier le cours mais encore de transformer durablement la relation de l'homme à luimême et au monde social, personnes et construits sociaux matériels et symboliques (Vygotski, 1930/1985). Vygotski propose ensuite une unité d'analyse plus fondamentalement sémiotique dont il examine l'une des formes emblématiques, la «signification du mot» qui permet d'approcher plus spécifiquement la dynamique de développement réciproque du langage et de la pensée (Vygotski, 1934/1997). Au plan méthodologique, cette deuxième théorisation élargit notablement la théorisation première, l'unité d'analyse de la signification permettant non seulement de considérer la conduite humaine dans l'ensemble de ses possibles constitutifs (intra- comme interindivuels, instrumentaux comme sémiotiques) mais encore d'envisager le principe de la dynamique développementale (Moro, 2000).

Contrairement à l'idée reçue, la médiation n'est jamais examinée par Vygotski que sous son acception intra-inviduelle et monologique (selon l'acception de Yakubinskii, in On dialogic speech, 1923, cité par Wertsch, 1985; voir aussi Moro, à paraître) focalisant prioritairement le pôle intra-individuel du développement, i.e. l'interaction du sujet avec l'outil et/ou avec le signe pour traquer les transformations successives de la pensée via l'élaboration corrélative de nouveaux instruments psychologiques (cf. la construction de la pensée conceptuelle et du langage). Le concept de médiation n'a jamais fait l'objet d'une articulation théorique avec la notion de Zoped (pôle interindividuel du développement) ces deux objets étant théorisés de manière discontinue au sein de la théorie vygotskienne. Toutefois, nous avons pu montrer que, sous son acception sémiotique, la médiation peut être étendue aux processus communicationnels (donc à la Zoped) et ainsi offrir la possibilité d'une théorisation unitaire des processus d'interaction sujet-sujet-objet (Moro, 2000; à paraître).

\section{La problématique des unités d'analyse}

L'une des grandes questions auxquelles se confrontent les approches socio-culturelles de la cognition est celle de la définition d'unités d'analyse permettant de rendre compte du fait psychologique et de sa constitution - question qui comme le soulignent très justement Lave \& Wenger (1991, p. 47) est absolument non problématique dans les approches individuo-centrées. Pour Vygotski dont la réflexion méthodologique constitue la référence en la matière (Vygotski, 1934/1997; voir aussi Moro, 2000; Moro \& Schneuwly, 1997; Zinchenko, 
1985), l'unité d'analyse joue le rôle d'indicateur du principe explicatif postulé au plan théorique en même temps qu'elle est en lien avec le découpage d'unités d'observation susceptibles d'objectiver le fait psychologique tant dans ses dimensions inter- qu'intrapsychologiques.

Si l'on considère les thèses de Lave et de Rogoff (qui illustreront notre propos dans cette rubrique), on constate la difficulté qu'il y a à formuler au plan théorique une unité qui articule à la fois les processus individuels et sociaux que Lave (1988) pourtant appelle de ses vœux. Lave et Rogoff avancent le concept de participation qui permet de ressaisir l'activité dans un cadre socio-culturel, lequel est censé prendre le relais du concept d'intériorisation, considéré comme trop mentaliste et accréditant une version transmissive et assimilatrice de l'apprentissage. Mais, comme le fait remarquer très justement Nelson $(1996)^{8}$, le processus critique se situe dans l'activité envisagée comme phénomène social externe et ne rend pas compte de l'activité psychique en propre (soit intra-individuelle).

Pour Lave, cette reconceptualisation s'inscrit principalement dans la filiation de la psychologie critique (Holzkamp, 1987) mais aussi dans la tradition de la psychologie de l'activité de Léontiev et de ses continuateurs comme Engeström, 1987 (in Lave \& Wenger, 1991). Rogoff revendique également cette dernière filiation, citant Léontiev, 1981 et Zinchenko, 1985 (in Rogoff, 1996) et également Engeström, 1993 (in Rogoff, 1998). Nombre de théoriciens de la Cognition située se réclament du modèle d'Engeström. Dans le modèle systémique de l'activité qu'il propose, la médiation instrumentale, dérivée de la conception vygotskienne, est resituée dans ses rapports à la communauté des autres personnes, aux règles sociales et à la division du travail dans une reformulation plus léontievienne. L'attractivité de ce modèle réside notamment dans le fait qu'il inclut la structure du monde social et permet de faire porter l'analyse sur la dynamique des relations entre personnes et notamment entre apprenants et experts dans le contexte d'une pratique partagée (Lave \& Wenger, 1991). Les limites de ce modèle est qu'il ne permet pas la saisie des processus intra-psychologiques et donc les processus de développement.

\section{La médiation dans le contexte de l'activité située/distribuée}

Dans les travaux de Lave et de Rogoff, la conception de la médiation apparaît plus particulièrement liée à l'analyse des processus communicationnels, comme le suggère le concept de "participation" auquel les objets d'apprentissage tendent à se confondre. Ainsi, l'accent porte-t-il davantage sur le processus de socialisation intrinsèque à l'acte d'apprendre (appropriation de normes, de valeurs, de modes d'être relatifs au savoir, processus de construction identitaire, etc.) que sur le processus effectif d'apprentissage du savoir en lui-même et des normes qui le constituent (voir la considération outre-Atlantique de la médiation dans les termes du social other en rapport avec la seule considération de la théorisation du 
concept de zone proximale du développement, cf. Van der Veer \& Valsiner, 1991; Moro \& Schneuwly, 1997).

Dans la conception de Hutchins, toute autre est la notion. Cette fois, l'accent est porté sur la tâche (à laquelle l'activité se trouve tendanciellement réduite) et en particulier sur les artefacts considérés comme des mondes médiatisant la tâche. La problématique de la médiation est ici étroitement liée à la tâche au sens où l'artefact transforme la tâche et en modifie cognitivement sa résolution. Hutchins (1997) indique: "Je considère que la médiation réfere à un mode particulier de comportement en rapport avec une tâche qui permet la coordination avec une structure médiatisante [artefact] qui n'est pas elle-même inhérente au domaine de la tâche». (p. 338, trad. pers.) La position instrumentale de Hutchins, même si elle peut être considérée comme une avancée par rapport à la conception seulement prothétique de l'artefact (à la seule fonction d'aide), n'est ainsi pas assimilable à la conception vygotskienne de l'instrument psychologique qui est synonyme de transformation durable des fonctions psychiques et de développement.

Cette dernière considération sur la médiation est en lien avec la finalité (ou objet) de l'activité. Si chez Hutchins, l'objet vers lequel l'action est dirigée est fortement lié à l'activité productive, chez Lave et chez Rogoff, cet objet se situe à mi-chemin entre l'activité productive et l'activité d'éducation-apprentissage, les deux finalités co-existant au sein de l'activité. Ce qui, ainsi que le reconnaissent Lave \& Wenger (1991), n'est pas sans avoir des incidences complexes «sur l'approfondissement et le changement de la compréhension pour chacun des membres de la communauté de pratiques» (p. 86, trad. pers.). Cette problématique n'est pas sans impact pour la réflexion sur le sens de l'apprentissage scolaire versus extra-scolaire, la préoccupation centrale de l'école étant la transformation des modes de penser (visée épistémique). D’où l'impérieuse nécessité de disposer d'une conception articulée des processus d'éducation/enseignement-apprentissage-développement pour une meilleure compréhension du développement au plan scolaire.

\section{La spécificité du développement en contexte scolaire versus extra-scolaire}

Il nous paraît utile d'entamer notre discussion en rappelant le présupposé de base de Vygotski que Schneuwly (1995) résume ainsi: «Le développement à l'âge scolaire n'est possible que grâce à l'enseignement; il présuppose cette forme d'éducation; les fonctions psychiques ne peuvent se développer que grâce à lui.» (p. 28ss) (souligné par nous) La forme d'éducation qui est pointée ici, «c'est l'enseignement disciplinaire, systématique, évitant la fausse concrétude [...].» (Schneuwly, 1995, p. 31) Cette considération contient en filigrane l'idée de rupture entre activité scolaire et extra-scolaire. Rupture que Vygotski thématise notamment au travers de la différence d'orientation entre le développement des concepts quotidiens et des concepts scientifiques ${ }^{9}$ (cf. Pensée et langage, 1934/1997, chapitre 6). 
"Le travail sur les concepts scientifiques en classe est axé essentiellement sur leur élaboration systématique à l'intérieur d'un système, sans référence nécessaire à l'expérience concrète de l'enfant, pour construire un usage non spontané.» (Schneuwly, 1995, p. 29)

Ainsi, pour Vygotski, dans le cadre scolaire, il s'agit de rompre avec les "dispositions à agir» acquises au cours des pratiques quotidiennes d'apprentissage et d'instaurer une forme de «suspension" par rapport à l'activité courante, propre à susciter la réflexion, la prise de conscience, bref le contrôle «dirigé» de sa propre activité psychique pour accéder à des systèmes de savoir de plus en plus complexes - lesquels requièrent un enseignement systématique qui va de pair avec l'objectivation maximale de ces savoirs. Cette suspension s'exprime dans le changement de direction imprimée à l'activité scolaire (si on la compare aux activités quotidiennes). ${ }^{10}$ Alors que l'activité en contexte extra-scolaire se trouve majoritairement régie par des finalités d'ordre pragmatique, dans le cadre scolaire, un changement fondamental de direction est imprimé à l'activité. Cette dernière (si on la compare aux activités quotidiennes) est alors explicitement dirigée vers l'acquisition de savoirs spécifiques, les tâches (activités à finalité pragmatique) étant dès lors surdéterminées par les objectifs d'apprentissage (activités à finalité épistémique). Ces diverses considérations entrent en résonance avec les propos de Bourdieu sur la Scholastic view. Citons Bourdieu (1994):

Scholastic view est une expression qu'Austin emploie en passant dans Sense and Sensibilia et dont il donne un exemple: l'usage particulier du langage qui, au lieu d'appréhender ou de mobiliser le sens d'un mot qui est immédiatement compatible avec la situation, recense tous les sens possibles de ce mot, en dehors de toute référence à la situation. Cet exemple, très significatif, enferme l'essentiel de ce qu'est la scholastic view. Il s'agit d'un point de vue tout à fait particulier sur le monde social, sur le langage ou tout autre objet de pensée, qui est rendu possible par la situation de skholè, de loisir, dont l'école - mot qui vient aussi de skholè - est une forme particulière, en tant que situation institutionnalisée de loisir studieux. L'adoption de ce point de vue scolastique est le droit d'entrée tacitement exigé par tous les champs savants: la disposition "neutralisante» (au sens de Husserl), impliquant la mise en suspens de toute thèse d'existence et de toute intention pratique, est la condition - autant au moins que la possession d'une compétence spécifique - de l'accès au musée et à l'auvre d'art. Elle est aussi la condition de l'exercice scolaire comme jeu gratuit, expérience mentale, qui est à elle-même sa fin (p. 221ss.).

Si chez Resnick, il s'agit d'inverser le cours des croyances spontanées qui entreraient en contradiction avec les conceptions antécédentes y compris biologiques (cf. supra, l'exemple de la constitution des concepts en physique), la nécessité de la rupture invoquée par Vygotski apparaît bien plus comme le fruit de l'orientation épistémologique du modèle où l'éducation et les construits sociaux sont considérés comme constitutifs du développement. Ainsi cette forme de mise en suspens de l'activité, associée à l'objectivation des savoirs conçus dès lors comme 
outils - que précisément Lave (1997) récuse - constitue chez Vygotski l'une des conditions pour accéder à l'apprentissage des savoirs complexes. Ce qui implique de rompre avec les logiques de divulgation-appropriation des savoirs dans les pratiques quotidiennes (cf. Lave, 1997). Et là, nous voudrions souligner le paradoxe qu'il y a chez Lave et chez Rogoff à mettre en avant la spécificité des communautés de pratiques (et donc des contextes institutionnels) et, dans le même temps, d'ériger l'apprenticeship en modèle pour l'apprentissage à l'école (cf. aussi Brown, Collins \& Duguid, 1989). Dans la logique même des propositions des théoriciens de la Cognition située, ne conviendrait-il pas d'insister sur ce qui est spécifique à l'institution scolaire au regard des autres communautés de pratiques, en particulier du point de vue des systèmes d'activités qui s'y déploient et ce, en fonction des finalités qui sont assignées à l'école?

\section{En guise de conclusion}

Au terme de ce bref parcours dans le champ de la Cognition située et du faisceau que nous avons braqué sur certaines de ses problématiques à l'aide du paradigme historico-culturel vygotskien, nous nous bornerons dans le cadre de cette conclusion à poser les termes d'un questionnement plus général que nous inspirent les positions ci-dessus évoquées. Comme le soulignent Kirshner \& Whitson (1997 a $\&$ b), les risques qui guettent les travaux sur la Cognition située sont directement proportionnels à l'engouement qu'ils ont suscité et qu'ils suscitent encore. Nous soulignerons ici certains des aspects qui, en tant que vygotskienne, nous alertent plus particulièrement.

En partant de l'analyse des situations (ou des tâches), les travaux sur la Cognition située dans le même temps qu'ils pointent le rôle constitutif du social et de la culture dans les apprentissages, tendanciellement font porter l'accent sur la dimension de flexibilité et d'adaptabilité du sujet eu égard aux différentes situations et aux exigences qu'elles comportent. C'est ce qui transparaît notamment chez Resnick (1994), qui considère qu' «apprendre, pour le rationaliste situé, consiste à s'accorder à la situation immédiate, en devenant bon dans la situation dans laquelle on agit. [...] Le cœur de l'argument résidant alors dans le fait qu'apprendre revient à passer au travers de situations successives dans lesquelles l'individu devient compétent» (p. 479, trad. pers.). Le lien avec la problématique des compétences dont Bronckart \& Dolz (1999) dénoncent le risque de dérive vers des positions utilitaristes est ici manifeste.

Cette appréciation est renforcée par le fait d'une centration quasi-exclusive sur des systèmes d'explication dont le noyau critique reste extérieur à l'individu et ne permet que très rarement de focaliser les mécanismes de construction de la pensée (cf. les constructs de "participation», de «communauté de pratiques», etc.) et la difficulté corrélative qu'il y a à promouvoir des modèles qui articulent véritablement perspective individuelle et perspective sociale (cf. supra, nos considé- 
rations sur les unités d'analyse du fonctionnement psychique). En témoignent, de façon corollaire, l'abandon explicite chez certains théoriciens de la Cognition située de la problématique de l'intériorisation (Lave \& Wenger, 1991) ainsi que de celle «des concepts et des représentations mentales [... en tant que ces derniers objets] sont essentiellement focalisés par la psychologie cognitive» (Kirshner \& Whitson, 1997b, trad. pers.). Nous considérons que la voie ouverte par Vygotski, qui propose une articulation générale entre les processus d'éducation/enseignement-apprentissage-développement et qui, ce faisant, (r)établit le rôle primordial des construits culturels (signes et outils) dans la formation du psychisme et de la conscience sans pour autant abandonner la problématique de l'intériorisation, offre une possibilité non négligeable d'éviter les différentes formes de réduction de la pensée alternativement aux seuls processus individuels ou aux seuls processus sociaux. De la même manière, la réflexion puissante que Vygotski développe sur la nécessité de formuler une perspective éducative forte en même temps que sa centration sur le développement de la personne apparaît comme une forme de contrepoids (sans être le seul) aux risques d'une désinstitutionnalisation de l'école que dénoncent Bronckart \& Dolz (1999) et Rochex (1999) notamment, mais également des théoriciens de la Cognition située comme Kirshner \& Whitson (1997a \& 1997b).

\section{Notes}

1 Nombre de réflexions au sein de cet article émanent de près ou de loin de mes échanges avec Bernard Schneuwly. Je tiens ici à le remercier tout particulièrement. Je voudrais également remercier Pierre-André Dupuis pour la lecture qu'il a faite de ce texte et pour ses précieuses remarques.

2 Le concept de participation, déjà invoqué par Lave \& Wenger (cf. supra), est également présent dans d'autres travaux comme ceux de White \& Siegel (1984). On en trouve déjà trace chez Dewey (1916): «L'environnement éducatif [...] est vraiment éducatif dans ses effets dans la mesure où un individu partage ou participe à quelqu'activité conjointe. En prenant part à l'activité associée, l'individu s'approprie le but qui l'anime, devient familier de ses méthodes et de ses contenus, acquiert les habiletés nécessaires, et s'imprègne de ses entours émotionnels» (p. 26) (trad. pers.).

3 En anthropologie, le concept d'artefact désigne toute chose qui porte la marque humaine et réfere en principe aux objets matériels fabriqués mais non exclusivement, Cole (1996) citant également, à titre d'artefact, le langage. Dans une acception culturelle, l'instrument désigne l'artefact tel qu'il se trouve utilisé en situation incluant alors le rapport au sujet. Quant à l'outil, il est susceptible des deux acceptions. Dans le cadre de notre revue, nous reprenons fidèlement la terminologie employée par les différents auteurs. Les indications de définition données ici leur sont relatives et n'ont d'autre but que de faciliter le travail du lecteur. Il convient ainsi de noter que les frontières entre ces différentes définitions sont particulièrement labiles et peuvent, selon les auteurs, révéler des options théoriques nuancées, parfois antagoniques. Outre certaines des références indiquées dans le présent texte, on pourra consulter Dolz, Moro \& Pollo (à paraître); Moro (1999, 2000); Rabardel (s.d.); Schneuwly \& Pfeiffer (à paraître); Vygotski (1930/1985). 
4 Citons Dewey (1938): «Dans l'expérience réelle, il n’y a jamais [...] d'objet ou d'événement singulier, un objet ou un événement est toujours une partie, une phase ou un aspect spécial d'un monde d'expérience - une situation» (1938, p. 67) (trad. pers.).

5 Chez Vygotski, seules les fonctions psychiques supérieures (i.e. à partir de l'avènement du langage) présentent la particularité d'être socialement médiatisées, les fonctions psychiques (dites) inférieures étant considérées comme biologiquement déterminées. Nous noterons que des travaux récents (Moro, 1999; Moro \& Rodriguez, en préparation; Rodriguez et Moro, 1999) attestent d'une médiation sociale des fonctions psychiques inférieures, i.e. concernant la période préalable à l'avènement du langage.

6 Si l'on se réfere à Schneuwly (1995, p. 16ss), il serait d'ailleurs plus juste de dire «enseignement-apprentissage» en lieu et place d'apprentissage pour être consistant avec le sens d'obuchenie, terme employé par Vygotski pour désigner le processus bi-directionnel de transmission-appropriation des connaissances.

7 Nous ne pouvons, dans le cadre de ces lignes, entrer dans une discussion approfondie du paradigme vygotskien (cf. Moro, 1999, p. 24-40; 2000). Reste qu'indéniablement la puissance programmatique du paradigme, dans ses élaborations méthodologiques et dans sa dernière mise en œuvre au plan de la théorie psychologique, fait que la théorie vygotskienne fournit à ce jour quelques-unes des propositions les plus stimulantes pour débrouiller l'écheveau complexe des relations entre éducation/enseignement-apprentissage et développement. Même si, pour nombre d'entre elles, ces propositions restent encore à élaborer (Clot, 1997).

8 La remarque de Nelson s'adresse à Rogoff mais les termes de son évaluation valent aussi pour Lave.

9 Les didacticiens parleraient aujourd'hui de concepts transposés. Mais, comme le signale Schneuwly (1995), "Vygotski ne disposait pas d'une théorie de la transposition didactique» qui lui aurait permis d'envisager un passage moins immédiat «du concept scientifique [...] intégré dans une pratique scientifique qui en définit la signification, au concept académique introduit en classe dans une pratique d'enseignement» (p. 29).

10 Le processus décrit par Vygotski se complexifie en raison du fait que les systèmes de savoir jouent eux-mêmes un rôle de guidage dans l'apprentissage (cf. notamment le 7 ème chapitre de Pensée et langage, Vygotski, 1934/1997; cf. aussi le concept d'usage tel que théorisé par Moro, 1999, pp. 122-126).

\section{Références bibliographiques}

Allal, L. (2000). Discussion. Et l'architecture des savoirs? Revue suisse des sciences de l'éducation, 22, (1), 165-172.

Anderson, J. R., Reder, L. M. \& Simon, H. A. (1997). Situated versus cognitive perspectives. Form versus substance. Educational Researcher, 26, (1), 18-21.

Berkowitz, M. W. \& Gibbs, J. C. (1985). The process of moral conflict resolution and moral development. In M. W. Berkowitz (Ed.), Peer conflict and psychological growth (pp. 71-84). San Francisco: Jossey Bass.

Bourdieu, P. (1994). Raisons Pratiques. Sur la théorie de l'action. Paris: Seuil.

Bronckart, J. P. \& Dolz, J. (1999). La notion de compétence: Quelle pertinence pour l'étude de l'apprentissage des actions langagières? In J. Dolz \& E. Ollagnier (Ed.), L'énigme de la compétence en éducation (Raisons Educatives No 1999/1-2/2, pp. 27-44). Bruxelles: DeBoeck.

Brown, J. S., Collins, A. \& Duguid, P. (1989). Situated cognition and the culture of learning. Educational Researcher, 18, (1), 32-42. 
Brown, A. L. \& Palincsar, A. S. (1989). Guided, cooperative learning and individidual knowledge acquisition. In L. B. Resnick (Ed.), Knowing, learning, and instruction (pp. 169180). Hillsdale, NJ: Erlbaum.

Bruner, J. (1990). Acts of meaning. Cambridge: Harvard University Press.

Clot, Y. (1997). Avant-Propos. In L. S. Vygotski, Pensée et Langage (pp. 7-18). Paris: La Dispute.

Cole, M. (1996). Cultural psychology. A once and future discipline. Cambridge, Massachusetts, London, England: The Belknap Press of Harvard University Press.

Dewey, J. (1913). L'enfant et l'école. Neuchâtel: Delachaux \& Niestlé.

Dewey, J. (1916). Democracy and education. New-York: Macmillan.

Dewey, J. (1938). Experience and Education. New-York: Macmillan.

Dolz, J., Moro, C. \& Pollo, A. (à paraître in Repères, No 22). Le débat régulé: de quelques outils et de leurs usages dans l'apprentissage.

Engeström, Y. (1987). Learning by expanding. Helsinki: Orienta-Konsultit Oy.

Engeström, Y. (1993). Developmental studies on work as a testbench of activity theory. In S. Chaiklin and J. Lave (Ed.), Understanding practice: Perspectives on activity and context (pp. 64-103). New-York: Cambridge University Press.

Forman, E. A. \& Cazden, C. B. (1985). Exploring Vygotskian perspectives in education: The cognitive value of peer interaction. In J. V. Wertsch (Ed.), Culture, communication and cognition: Vygotskian perspectives (pp. 323-347). Cambridge, UK: Cambridge University Press.

Forman, E., Minick, N. \& Stone, C. A. (Ed.). (1993). Contexts for learning: Sociocultural dynamics in children's development. New-York: Oxford University Press.

Greeno, J. F., Moore, J. L. \& Smith, D. R. (1993). Transfer of situated learning. In. D. K. Detterman \& R.J. Sterberg (Ed.), Transfer on trial: Intelligence, cognition, and instruction (pp. 99-167). Norwood, NJ: Ablex Publishing Corporation.

Griffin, P. \& Cole, M. (1984). Current activity for the future: The Zo-ped. In B. Rogoff \& J.V. Wertsch (Eds.). Children's learning in the "zone of proximal development» (pp. 45-64). San Francisco: Jossey-Bass.

Hanks, F. (1991). Foreword. In J. Lave \& E. Wenger, Situated learning. Legitimate peripheral participation (pp. 13-24). Cambridge, MA: Cambridge University Press.

Holzkamp, K. (1987). Critical psychology overcoming of scientific indeterminacy in psychological theorizing. In R. Hogan \& W. H. Jones (Ed.), Perspectives in personality (pp. 7589). Greenwich, CT: JAI Press.

Hutchins, E. (1990). The technology of team navigation. In J. Galegher, R. E. Kraut, \& C. Egido (Ed.), Intellectual teamwork: Social and technological foundations of cooperative work (pp. 191-120). Hillsdale NJ.: Erlbaum.

Hutchins, E. (1991). The social organization of distributed cognition. In L. B. Resnick, J. M. Levine, \& S. D. Teasley (Ed.), Perspectives on socially shared cognition (pp. 283-307). Washington, DC.: American Psychological Association.

Hutchins, E. (1993). Learning to navigate. In S. Chaiklin \& J. Lave (Ed.), Understanding practice. Perspectives on activity and context (pp. 35-63). New-York: Cambridge University Press.

Hutchins, E. (1995). Cognition in the wild. Cambridge, Mass.: MIT Press.

Hutchins, E. (1997). Mediation and automatization. In M. Cole, Y. Engeström \& O. Vasquez (Ed.), Mind, culture, and activity. Seminal papers from the Laboratory of Comparative $\mathrm{Hu}$ man Cognition (pp. 338-353). New-York: Cambridge University Press.

Johnson, W. \& Johnson R. T. (1989). Cooperation and competition: Theory and research. Edina, $\mathrm{MN}$ : Interaction.

Kirshner, D. \& Whitson, J. A. (Ed.). (1997a). Situated Cognition. Social, semiotic, and psychological Perspectives. Mahwah, New Jersey: Lawrence Erlbaum Associates. 
Kirshner, D. \& Whitson, J. A. (1997b). Obstacles to understanding cognition as situated. Educational Researcher, 27 (8), 22-28.

Lave, J. (1977a). Tailor-made experiments and evaluation the intellectual consequences of apprenticeship training. Quarterly Newsletters of the Laboratory of Comparative Human Cognition, 1, 1-3.

Lave, J. (1977b). Cognitive consequences of traditional apprenticeship training in West Africa. Anthopology and Education Quarterly, 8, 177-180.

Lave, J. (1988). Cognition in practice. Cambridge, UK: Cambridge University Press.

Lave, J. (1991). Situated learning in communities of practice. In L. B. Resnick, J. M. Levine \& S. D. Teasley (Ed.), Perspectives on socially shared cognition (pp. 63-82). Washington, DC: American Psychological Association.

Lave, J. (1997). The culture of acquisition and the practice of understanding. In D. Kirshner \& J. A. Whitson (Ed.), Situated Cognition. Social, semiotic, and psychological Perspectives (pp. 17-36). Mahwah, New Jersey: Lawrence Erlbaum Associates.

Lave, J. \& Wenger, E. (1991). Situated learning: legitimate peripheral participation. Cambridge, UK: Cambridge University Press.

Leontiev, A. N. (1981). The problem of activity in psychology. In J. V. Wertsch (Ed.), The concept of activity in Soviet psychology (pp. 37-71). Armonk, NY: Sharpe.

Mead, G. H. (1934). Mind, self and society from the standpoint of a social behaviorist. Chicago: University of Chicago Press.

Moro, C. (1999). L'usage de l'objet comme experimentum crucis pour l'analyse des significations durant la période préverbale. Etude longitudinale chez le bébé entre 7 et 13 mois. Thèse de doctorat es sciences de l'éducation et es psychologie. Universités de Genève et de Bordeaux 2.

Moro, C. (2000). Vers une approche sémiotique intégrée du développement humain. Note de synthèse pour l' Habilitation à diriger des recherches. Université Bordeaux 2.

Moro, C. (à paraître). Médiation et développement. Enjeux et perspectives de la théorie de L. S. Vygotski. In M. Zulauf \& M. Wirthner (Ed.), Ouvrage inspiré des "Journées francophones de recherches en éducation musicale» "Quelles théories du développement musical chez l'enfant?" Neuchâtel, 26-27 avril 1999; "Quelles médiations pour le développement musical de l'enfant?» Neuchâtel, 7-8 février 2000. Paris: L'Harmattan.

Moro, C. \& Rodriguez, C. (en préparation pour Delachaux \& Niestlé). Le bébé et le signe. Vers la définition d'une approche sémiotique du développement humain [titre provisoire].

Moro, C. \& Schneuwly, B. (1997). L'outil et le signe dans l'approche du fonctionnement psychologique. Introduction. In C. Moro, B. Schneuwly \& M. Brossard (Ed.), Outils et signes. Perspectives actuelles de la théorie de Vygotski (pp. 1-17). Berne: Peter Lang.

Mugny, G., Perret-Clermont, A.-N. \& Doise, W. (1981). Interpersonal coordinations and social differences in the construction of the intellect. In G. M. Stephenson \& J. M. Davis (Ed.), Progress in applied psychology, Vol. 1 (pp. 315-343). New-York: Wiley.

Nelson, K. (1996). Language in cognitive development. The emergence of the mediated mind. Cambridge: Cambridge University Press.

Norman, D. A. (1988). The psychology of everyday things. New-York: Basic Books.

Norman, D. A. (1993). Les artefacts cognitifs, Raisons Pratiques, 4, 15-34.

Packer, M. J. (1993). Away from internalization. In E. A. Forman, N. Minick \& C. A. Stone (Ed.), Contexts for learning (pp. 254-265). New-York: Oxford University Press.

Pea, R. (1991). Series Foreword. In J. Lave \& E. Wenger, Situated learning. Legitimate peripheral participation (pp. 11-12). Cambridge: Cambridge University Press.

Pea , R. (1993). Practices of distributed intelligence and designs for education. In G. Salomon (Ed.), Distributed cognitions: psychological and educational considerations (pp. 47-87). NewYork: Cambridge University Press.

Perkins, D. N. (1995). L'individu plus, une vision distribuée de la pensée et de l'apprentissage. Revue française de pédagogie, 111, 57-71. 
Rabardel, P. (s.d.). Les activités avec instruments. Note de synthèse pour l'Habilitation à diriger des recherches. Paris: Université Paris VIII.

Resnick, L. B. (1987). Learning in school and out. Educational Researcher, 16 (9), 13-20.

Resnick, L. B. (1994). Situated rationalism: Biological and Social Preparation for learning. In L. A. Hirchfeld \& S. A. Gelman (Ed.), Mapping the mind: Domain specificity in cognition and culture (pp. 474-493). Cambridge: Cambridge University Press.

Rochex, J. Y. (1999). Expérience scolaire et procès de subjectivation. L'élève et ses milieux. Note de synthèse pour l'Habilitation à diriger des recherches. Paris: Université Paris VIII.

Rodriguez, C. \& Moro, C. (1999). El mágico número tres. Cuando los niños aún no hablan. Barcelona: Paidós.

Rogoff, B. (1990). Apprenticeship in thinking: Cognitive development in social context. NewYork: Oxford University Press.

Rogoff, B. (1995). Observing sociocultural activity on three planes: Participatory appropriation, guided participation, and apprenticeship. In J. V. Wertsch, P. del Rio \& A. Alvarez (Ed.), Sociocultural studies of mind (pp. 139-164). Cambridge: Cambridge University Press.

Rogoff, B. (1996). Developmental transitions in children's participation in sociocultural activities. In A. J. Sameroff \& M. M. Haith (Ed.), The five to seven year shift. The age of reason and responsibility (pp. 273-294). Chicago: University of Chicago.

Rogoff, B. (1998). Cognition as a collaborative process. In D. Kuhn \& R. S. Siegler (Vol. Ed.), Handbook of child psychology, Vol. 2: Cognition, perception, and language (pp. 670-744). New-York: Wiley.

Rogoff, B. \& Toma, C. (1997). Shared thinking: community and institutionnal variations. Discourses Processes, 23, 471-497.

Rumelhart, D. E. \& McClelland, J. L. (1986). Parallel distributed processing: Explorations in microstructure of cognition. Vol. 1, Foundations. Cambridge, M.A.: Bradford Books.

Salomon, G., Perkins, D. N. \& Globerson, T. (1991). Partners in cognition: Extending human intelligence with intelligent technologies. Educational Researcher, 20, (3), 2-9.

Schneuwly, B. (1995). De l'importance de l'enseignement pour le développement Vygotski et l'école. Psychologie et Education, 21, 25-37.

Schneuwly, B. (1999). Le développement du concept de développement chez Vygotski. In Y. Clot (Ed.), Avec Vygotski (pp. 267-280). Paris: La Dispute.

Schneuwly, B. \& Pfeiffer, V. (à paraître in Repères, No 22). Les outils de l'enseignant. Un essai didactique.

Schwartz, Y. (2001). Théories de l'action ou rencontres de l'activité? In J.-M. Baudouin \& J. Friedrich (Ed.), Théories de l'action et éducation. Raisons Educatives 2001 (pp. 93-112). Bruxelles: De Boeck.

Valsiner, J. \& Van der Veer, R. (1993). The encoding of distance: The concept of the zone of proximal development and its interpretations. In R. R. Cocking \& K. A. Renninger (Ed.), The development of meaning of psychological distance (pp. 35-62). Hillsdale, NJ: Erlbaum.

Van der Veer, R. \& Valsiner, J. (1991). Understanding Vygotsky. The quest for a synthesis. Oxford: Blackwell.

Vygotski, L. S. (1930/1985). La méthode instrumentale en psychologie. In B. Schneuwly \& J. P. Bronckart (Ed.), Vygotski aujourd'hui (pp. 39-47). Neuchâtel-Paris: Delachaux \& Niestlé.

Vygotski, L. S. (1934/1997). Pensée et Langage. Paris: La Dispute.

Wertsch, J. V. (1985). Vygotsky and the social formation of mind. Cambridge: Harvard University Press.

White, S. H. \& Siegel, A. W. (1984). Cognitive development in time and space. In B. Rogoff \& J. Lave (Ed.), Everyday cognition: Its development in social context (pp. 238-277). Cambridge, M. A.: Harvard University Press.

Wood, D., Bruner, J. \& Ross, G. (1976). The role of tutoring in problem-solving. Journal of child psychology and psychiatry, 66, 181-191. 
Yakubinskii, L. P. (1923). O dialogicheskoi rechi [On dialogic speech]. Petrograd: Trudy Foneticheskogo Instituta Prakticheskogo Izucheniya Yazykov.

Zinchenko, V. P. (1985). Vygotsky's ideas about units of analysis of mind. In J. V. Wertsch (Ed.), Culture, communication and cognition (pp. 94-118). New-York: Cambridge University Press.

\section{Situierte Kognition und das historisch-kulturelle Paradigma von Wygotski: zentrale Probleme und Fragestellungen}

\section{Zusammenfassung}

Ziel dieses Aufsatzes ist es, einige Felder der theoretischen Diskussion zu beleuchten, die im Zusammenhang der «situated cognition» entstanden sind. Zu diesem Zweck untersucht die Autorin in einem ersten Schritt Texte, die von den Pionieren dieser Tradition publiziert worden sind, und versucht dabei die theoretischen Ausrichtungen, die sich in diesen Texten abzeichnen, herauszuheben. In einem zweiten Schritt analysiert sie anhand einiger Schlüsselthemen den Grad der Übereinstimmung zwischen den Zielen zweier theoretischer Felder, nämlich des «situated cognition» und des historisch-kulturellen Paradigmas im Gefolge Wygotskis.

\section{Cognizione situata e paradigma storico-culturale vygotskiano: problemi e interrogativi}

\section{Riassunto}

Questo contributo intende mettere in evidenza alcuni spazi di discussione teorica così come emergono dalle ricerche sulla cognizione situata. A tale scopo l'autrice inizia con un sondaggio attraverso i lavori dei pionieri di questo approccio e cerca di metterne a fuoco le opzioni teoriche. In seguito esamina sulla base di tre tematiche chiave (la problematica delle unità d'analisi, la definizione della mediazione e la specificità delle attività scolastiche in contrapposizione a quelle extra-scolastiche) la relazione esistente tra gli obiettivi dei due paradigmi teorici in questione: quello vygotskiano e quello che si ispira alla cognizione situata. 


\section{Contextual Cognition and the Historical-Cultural paradigm of Vygotsky: Central Order of Problems and Questions}

\footnotetext{
Abstract

The aim of this article is to first highlight some of the main theoretical aspects which underlie the conceptions of the contextual cognition amongst the pioneers of this tradition. Secondly, three key theoretical themes focused on by these contextual cognitionists are specifically analyzed: the question of the units of analysis, which is intrinsically linked to the social nature of cognition and learning; the different ways to define the processes of mediation in the process of activities; the theorization of school activities versus everyday activities and how these two kinds of activities are or are not related. Through these themes, a comparison of the theoretical options of both contextual cognition and the Vygotskian framework is proposed.
} 